\title{
Proteic boost enhances humoral response induced by DNA vaccination with the dnaK gene of Chlamydophila abortus but fails to protect pregnant mice against a virulence challenge
}

\author{
Céline HÉchard, Olivier GréPINET*, Annie Rodolakis \\ Unité de Pathologie Infectieuse et Immunologie, INRA-Centre de Tours, 37380 Nouzilly, France
}

(Received 21 March 2002; accepted 5 July 2002)

\begin{abstract}
In order to enhance the quantity and the protective properties of the antibodies induced by DNA vaccination with the heat shock protein dnaK gene of Chlamydophila abortus AB7 as well as to elicit an efficient cellular immune response, we vaccinated mice with a DNA prime followed by a boost with the recombinant DnaK protein. In non-pregnant mice, this strategy induced the same predominance of the IgG2a isotype as DNA immunization alone with a substantial increased antibody level. The induced antibodies had no in vitro neutralizing properties on C. abortus infectivity. Moreover, the proteic boost probably failed to elicit an efficient cellular immune response since the pregnant or non-pregnant mice were not protected against the bacterial challenge.
\end{abstract}

DNA immunization / proteic boost / Chlamydophila abortus / DnaK / Hsp70

\section{INTRODUCTION}

DNA vaccination represents a recent approach for the prevention of infectious diseases. DNA plasmids encoding for antigens are used to directly transfect the animal cells in situ and the antigen expression induces both cellular and humoral immune responses which are particularly appropriate for preventing intracellular bacterial infections [5]. DNA immunization appears to be a potential strategy for vaccine development against Chlamydiaceae [1].
Chlamydiaceae are Gram-negative obligate intracellular bacterial pathogens that cause a broad range of diseases in humans and animals [6]. DNA vaccination has already been used to elicit protective immune responses against Chlamydia trachomatis, Chlamydophila pneumoniae and Chlamydophila psittaci $[10,15,16]$. In preliminary studies, a DNA vaccine encoding the heat shock protein (hsp) DnaK (also called Hsp70) of Chlamydophila abortus induced a humoral response with an $\mathrm{IgG} 2 \mathrm{a}$ predominant isotype [8]. However, it

*Correspondence and reprints

Tel.: (33) 2474278 63; fax: (33) 2474277 79; e-mail: grepinet@tours.inra.fr 
failed to protect pregnant and non-pregnant mice against bacterial challenge probably because this dnaK DNA vaccine induces a too weak immune response. Difficulties in inducing a sufficient protective antibody level and cellular immune response with DNA immunization alone have led to methods that use DNA vaccination to prime and a recombinant protein to boost the immune system [4].

In the present study, we used such a prime-boost vaccination protocol in order to enhance the immune response induced by the dnaK DNA vaccine. Despite the increased humoral immune response, no difference in terms of protection was observed after the immunization with the dnaK DNA prime and the proteic boost or with the dnaK DNA vaccine alone in pregnant or non-pregnant mice.

\section{MATERIALS AND METHODS}

\subsection{C. abortus strains}

The virulent $C$. abortus AB7 ovine abortion strain isolated from an ovine abortion [7] and the vaccinal 1B strain obtained by selection of a temperature-sensitive mutant after nitrosoguanidine mutagenesis of C. abortus AB7 [12] were used. Bacteria were propagated in the yolk sac of embryonated SPF chicken eggs inoculated at day 7 , purified as previously described [3] and stored at $-70{ }^{\circ} \mathrm{C}$.

\subsection{Plasmid construction and recombinant DnaK protein}

In order to obtain the vaccinal vector, the dnaK gene of C. abortus AB7 (GenBank AF384685 accession number) was inserted into the pcDNA3.1 (Invitrogen, San Diego, CA, USA) eukaryotic expression vector to generate pcDNA3.1::DnaK [8]. The recombinant DnaK protein was produced as previously described [8].

\subsection{Immunization and challenge infection}

All the studies were done on six-weekold female outbred OF1 Swiss mice (IFFA Credo, L'Arbresle, France). For abortion tests, five groups of 20 mice were made. Prior to DNA immunization, each mouse was injected with cardiotoxin (Latoxan, Valence, France) into the tibialis anterior muscles of both hind legs. At days 0 and 21 , the mice were anesthetized by intraperitoneal injection of ketamine and xylazine ( 80 and $8 \mathrm{mg} / \mathrm{kg}$ of body weight, respectively) and immunized with pcDNA3.1 or pcDNA3.1::DnaK plasmids by intramuscular injections $(50 \mu \mathrm{g}$ in each tibialis anterior). At day 42, the control mice received a third injection of pcDNA3.1 and the vaccinated mice were boosted with a subcutaneous intradorsal injection of $10 \mu \mathrm{g}$ of the recombinant DnaK protein emulsified in Freund incomplete adjuvant (Sigma, Saint Louis, MO, USA). Mice were mated at day 44 and challenged at day 58 by an intraperitoneal injection of $2 \times 10^{5}$ plaque forming units (pfu) of C. abortus AB7.

Additional control groups were done. First, a group of vaccinated control mice was obtained after one injection of $10^{5} 1 \mathrm{~B}$ vaccine bacteria at day 1 . The mice belonging to the virulence control group were infected but not immunized. Finally, mice of the pregnancy group were neither immunized nor challenged. The mice were considered protected when the number of living offspring per litter 8 days after birth was significantly different $(P<0.05)$ from the number of the control group as previously described [12].

Four groups of 16 non-pregnant mice were made for immunological trials. As for the mice in the abortion test, these non-pregnant mice were immunized with the 1B vaccine, pcDNA3.1 or pcDNA3.1::DnaK/DnaK boost. One additional group was immunized by PBS (same quantity, site and time as the DNA 
injections) and consequently served as the virulence control group.

\subsection{Antibody response}

The specificity of anti-DnaK antibodies was checked by an ELISA test and a western blot as previously described [8]. The determination of antibody isotypes was performed with the pooled sera of pcDNA3.1::DnaK- or pcDNA3.1::DnaK/ DnaK boost-vaccinated mice collected at day 56 [8]. The in vitro neutralization assay was done by the plaque reduction assay [8].

\subsection{Chlamydial enumeration in the organs}

Ten non-pregnant mice were immunized with $\mathrm{PBS}$, the $1 \mathrm{~B}$ vaccine, the pcDNA3.1 or the pcDNA3.1::DnaK/DnaK boost as described above. Five days after challenge, the mice were euthanized and the spleens were aseptically removed, weighed and frozen at $-80^{\circ} \mathrm{C}$.

Five pregnant mice of the groups described above were euthanized at day 63. The uterus and the spleen were aseptically removed, all the placentas from the same uterine horn were dissected from the fetuses and pooled. The pooled placentas and the spleens were weighed and frozen at $-80{ }^{\circ} \mathrm{C}$. Organs were titrated by plaque assay on McCoy cells [13].

\subsection{Statistical tests}

The analysis of the results was performed using the InStat 2.03 software for Macintosh. For all protective results, the mean was calculated using a one way analysis of variance, and a comparison of the means was then carried out through a Student-Newman-Keuls multiple comparison test. The minimal statistical significance was judged at $P<0.05$.

\section{RESULTS}

\subsection{Anti-DnaK antibody response to DNA vaccination and proteic boost}

No anti-DnaK specific antibodies were detected in the sera of mice vaccinated with the pcDNA3.1 control plasmid. AntiDnaK specific antibodies were detected in the sera collected at days 56 and 63 from the mice vaccinated with pcDNA3.1:: DnaK/DnaK boost (Fig. 1). These sera as well as the anti-DnaK monoclonal antibody (MAb) 18.1 recognize DnaK in the C. abortus AB7 extract (Fig. 1B). Then, the pcDNA3.1::DnaK/DnaK boost vaccination of mice induced specific antibodies directed against the DnaK protein of C. abortus AB7.

In order to quantify the anti-DnaK humoral response, the sera from immunized mice were collected 20 days after each DNA (days 19 and 41) and protein injection (day 56) as well as 5 days after the chlamydial challenge (day 63). No DnaK-specific IgG antibodies measured by ELISA were identified in sera from non-immunized mice or from mice immunized with pcDNA3.1 (data not shown). The quantity of IgG anti-DnaK antibodies was comparable in the sera of pcDNA3.1::DnaK- or pcDNA3.1::DnaK/ DnaK boost-immunized mice after two injections of DNA (day 41). Nevertheless, it increased in a higher way after the proteic boost than after the third DNA injection (respectively 7700 and 1450 arbitrary units at day 56). Thus, the proteic boost significantly enhanced $(P<0.01)$ the antibody response.

Moreover, the DNA/proteic boost immunization induced the same predominance of the $\operatorname{IgG} 2 \mathrm{a}$ antibody subclass than DNA vaccination alone and the pcDNA3.1::DnaK/DnaK boost-vaccinated mice sera had no in vitro neutralizing effect on C. abortus infectivity (data not shown). 


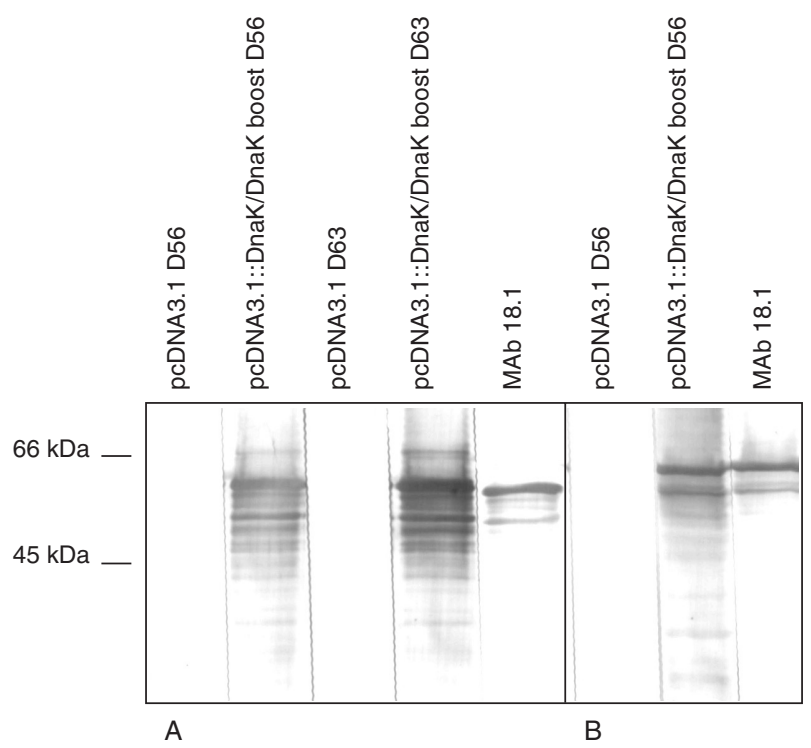

Figure 1. Specificity of the sera of pcDNA3.1- or pcDNA3.1::DnaK/DnaK boost-vaccinated mice analyzed by immunoblotting. Mice were immunized at days 0,21 and 42 and challenged at day 58 with $C$. abortus AB7. The sera collected at days 56 and 63 were tested by western blot using the purified recombinant DnaK protein $(0.1 \mu \mathrm{g} / \mathrm{lane})(\mathrm{A})$ or $C$. abortus AB7 extracts $\left(8.10^{6}\right.$ bacteria/ lane) (B) as antigens. Molecular sizes are given in the left hand margin.

\subsection{Protection against $C$. abortus AB7 challenge}

The number of live newborn mice in pcDNA3.1- and pcDNA3.1::DnaK/DnaK boost-vaccinated mice was significantly lower $(P<0.05)$ than in the $1 \mathrm{~B}$-vaccinated group and significantly higher $(P<0.05)$ than in the virulence group (Fig. 2). Thus, pcDNA3.1- and pcDNA3.1::DnaK/DnaK boost-vaccinated mice were partially protected from the C. abortus AB7 challenge. Since chlamydial titres of pcDNA3.1- and pcDNA3.1::DnaK/DnaK boost-vaccinated mice placentas did not show significant difference with titres of the virulence group, DNA vaccination induced no placental protection in pregnant mice (Fig. 3).

Chlamydial titres of the pcDNA3.1vaccinated mice spleens did not show a significant difference with the titres of the virulence group in pregnant mice (Fig. 4).
Nevertheless, the pcDNA3.1 control vaccine had a protective effect in nonpregnant mice. The titres of the pcDNA3.1::DnaK/DnaK boost-vaccinated mice did not show a significant difference with the titres of the virulence group in pregnant or non-pregnant mice. Therefore, DNA vaccination with the pcDNA3.1 control plasmid induced a non-specific splenic protection in non-pregnant mice and the dnaK DNA prime/proteic boost induced no splenic protection in pregnant and non-pregnant mice.

\section{DISCUSSION}

We previously showed that DNA vaccination with the hsp dnaK gene of $C$. abortus $\mathrm{AB} 7$ induces a non-protective humoral response with an anti-DnaK specific IgG2a predominant isotype [8]. In order to enhance the quantity and the neutralizing 


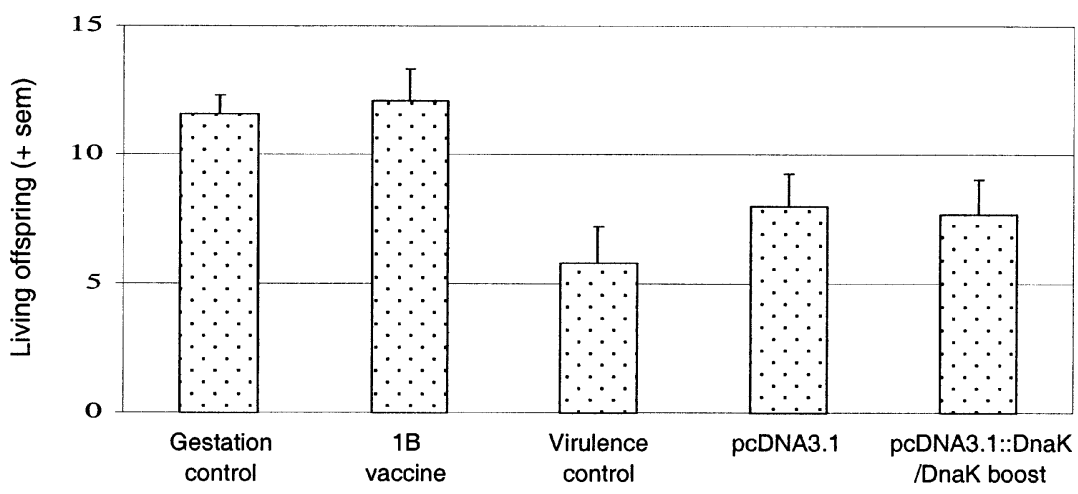

Figure 2. Effect of the DNA prime-proteic boost vaccination on viable offspring. The 1B vaccine, pcDNA3.1 and pcDNA3.1::DnaK/DnaK boost groups were respectively immunized with the live $1 \mathrm{~B}$ vaccine, the pcDNA3.1 plasmid and the pcDNA3.1::DnaK plasmid followed by a boost with the recombinant DnaK protein. The gestation control group was neither infected nor immunized and the virulence group was infected but not immunized. After immunization, the groups were infected at 12 days of gestation. The results were expressed as an average number of living offspring 1 week after birth $(+$ sem).

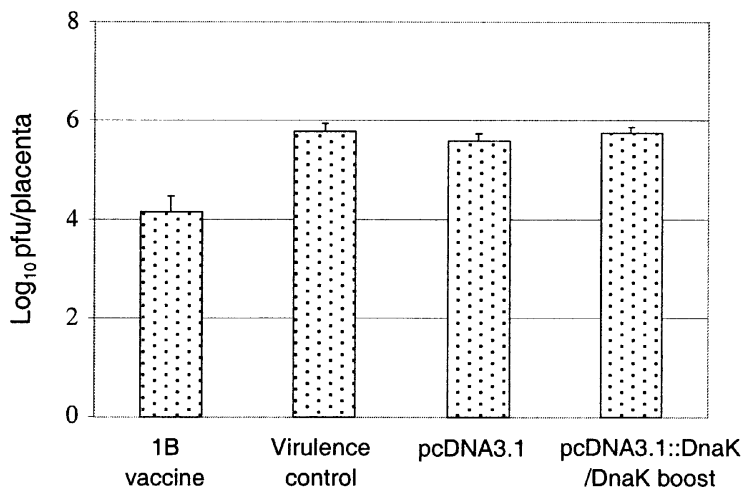

Figure 3. Chlamydial clearance in the placentas of pregnant mice. Mice were immunized and mated, then they were challenged with $\mathrm{C}$. abortus $\mathrm{AB} 7$ and euthanized 5 days after the challenge. The quantities of bacteria in the placentas are expressed in $\log _{10}$ pfu per placenta $(+\mathrm{sem})$.

activity of the antibodies as well as the cytotoxic T-lymphocyte response, we used a DNA prime and a proteic boost as a vaccination strategy [4]. The IgG2a isotype raised after DNA priming was preserved by a DNA/protein boost immunization. The induction of the predominance of the $\mathrm{IgG} 1$ or IgG2a isotype is determined by the type of priming vaccine [14]. DNA immu- nization usually elicits the predominance of the IgG2a isotype while the recombinant protein vaccination preferentially induces the IgG1 isotype. Although the proteic boost induced a stronger humoral response, the antibodies induced by the pcDNA3.1::DnaK/DnaK boost immunization had no neutralizing effect. So, the lack of protection observed after the DNA 


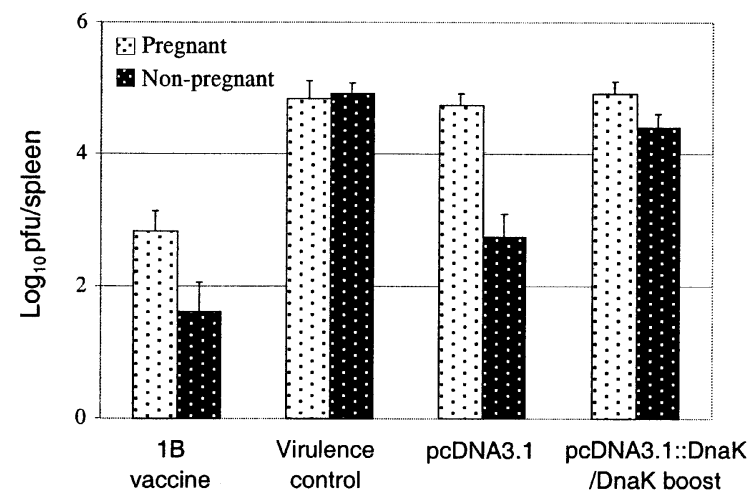

Figure 4. Chlamydial clearance in the spleens of pregnant or non-pregnant mice. Mice were immunized, then they were challenged with $C$. abortus AB7 and euthanized 5 days after the challenge. The quantities of bacteria in the spleens are expressed in $\log _{10}$ pfu per spleen $(+\mathrm{sem})$.

vaccination with the dnaK gene alone was probably due to the weak neutralizing properties of the antibodies rather than to a weak quantity as previously expected [8]. This absence of a neutralizing effect could be due to the inaccessibility of the antigen on the cell surface. Indeed, a recent report suggests that Hsp70 is not a surface displayed ligand on purified elementary bodies and needs a partial reduction of the cell wall to be exposed [11].

This lack of a neutralizing effect was confirmed by the evaluation of the protection induced by pcDNA3.1::DnaK/DnaK boost vaccination after a chlamydial challenge. As we observed during DNA immunization with the $d n a K$ gene alone [8], only a partial and non-specific protection was shown on living offspring in pcDNA3.1as well as in pcDNA3.1::DnaK/DnaK boost-vaccinated mice. This effect can possibly be explained by the immunostimulating properties of the $\mathrm{CpG}$ motifs present in bacterial DNA [9].

In a mouse model of $C$. abortus infection, the cellular immune response was more efficient than the humoral immune response in decreasing systemic infection [2]. However, we could not extensively analyze the cellular immune response induced by immunization because the OF1 outbred mouse model used in this experiment was not suitable for such studies. Since no protection was observed neither in splenic and placental localization in pregnant mice nor in splenic localization in non-pregnant mice, the pcDNA3.1::DnaK/ DnaK boost probably failed to induce a protective cellular immune response. Moreover, no DTH response was observed in mice immunized with pcDNA3.1::DnaK alone [8].

Despite the increased level of IgG2a antibodies induced by the DNA prime/proteic boost, no protection was observed against the $C$. abortus challenge in pregnant and non-pregnant mice. Therefore, this vaccination strategy failed to enhance the protective properties of the elicited antibodies and to induce an efficient cellular immune response which remains the main actor of chlamydial infection resolution.

\section{ACKNOWLEDGMENTS}

The authors would like to thank Françoise Bernard for excellent technical assistance and Dr. S. Birkelund (University of Aarhus, Denmark) for the kind gift of the anti-DnaK monoclonal antibody 18.1. Céline Héchard was 
supported by a grant from INRA-Région Centre.

\section{REFERENCES}

[1] Brunham R.C., Zhang D.J., Yang X., McClarty G.M., The potential for vaccine development against chlamydial infection and disease, J. Infect. Dis. 181 Suppl. 3 (2000) S538-S543.

[2] Buzoni-Gatel D., Rodolakis A., Plommet M., $\mathrm{T}$ cell mediated and humoral immunity in a mouse Chlamydia psittaci systemic infection, Res. Vet. Sci. 43 (1987) 59-63.

[3] Caldwell H.D., Kromhout J., Schachter J., Purification and partial characterization of the major outer membrane protein of Chlamydia trachomatis, Infect. Immun. 31 (1981) 1161-1176.

[4] Dong-Ji Z., Yang X., Shen C., Lu H., Murdin A., Brunham R.C., Priming with Chlamydia trachomatis major outer membrane protein (MOMP) DNA followed by MOMP ISCOM boosting enhances protection and is associated with increased immunoglobulin $\mathrm{A}$ and Th1 cellular immune responses, Infect. Immun. 68 (2000) 3074-3078.

[5] Donnelly J.J., Ulmer J.B., Shiver J.W., Liu M.A., DNA vaccines, Annu. Rev. Immunol. 15 (1997) 617-648.

[6] Everett K.D.E., Chlamydia and Chlamydiales: more than meets the eye, Vet. Microbiol. 75 (2000) 109-126.

[7] Faye P., Charton A., Mage C., Bernard C., Le Layec C., Propriétés hémagglutinantes du "virus" de l'avortement enzootique des petits ruminants (souches de "Rakeia" d'origine ovine et caprine), Note préliminaire, Bull. Acad. Vét. Fr. 45 (1972) 169-173.

[8] Héchard C., Grépinet O., Rodolakis A., Protection evaluation against Chlamydophila abortus challenge by DNA vaccination with a dnaK-encoding plasmid in pregnant and non-pregnant mice, Vet. Res. 33 (2002) 313326.

[9] Krieg A.M., Immune effects and mechanisms of action of CpG motifs, Vaccine 19 (2001) 618-622.

[10] Penttilä T., Vuola J.M., Puurula V., Anttila M., Sarvas M., Rautonen N., Mäkelä P.H., Puolakkainen M., Immunity to Chlamydia pneumoniae induced by vaccination with DNA vectors expressing a cytoplasmic protein (Hsp60) or outer membrane proteins (MOMP and Omp2), Vaccine 19 (2001) 1256-1265.

[11] Raulston J.E., Davis C.H., Paul T.R., Hobbs J.D., Wyrick P.B., Surface accessibility of the 70-kilodalton Chlamydia trachomatis heat shock protein following reduction of outer membrane protein disulfide bonds, Infect. Immun. 70 (2002) 535-543.

[12] Rodolakis A., In vitro and in vivo properties of chemically induced temperature-sensitive mutants of Chlamydia psittaci var. ovis: screening in a murine model, Infect. Immun. 42 (1983) 525-530.

[13] Rodolakis A., Chancerelle L., [Plaque assay for Chlamydia psittaci in tissue samples (in French)], Ann. Inst. Pasteur Microbiol. B 128 (1977) 81-85.

[14] Sin J.I., Bagarazzi M., Pachuk C., Weiner D.B., DNA priming-protein boosting enhances both antigen-specific antibody and Th1type cellular immune responses in a murine herpes simplex virus-2 gD vaccine model, DNA Cell Biol. 18 (1999) 771-779.

[15] Vanrompay D., Cox E., Vandenbussche F., Volckaert G., Goddeeris B., Protection of turkeys against Chlamydia psittaci challenge by gene gun-based DNA immunizations, Vaccine 17 (1999) 2628-2635.

[16] Zhang D.-J., Yang X., Berry J., Shen C., McClarty G., Brunham R.C., DNA vaccination with the major outer-membrane protein gene induces acquired immunity to Chlamydia trachomatis (mouse pneumonitis) infection, J. Infect. Dis. 176 (1997) 1035-1040. 
